Check for updates

Cite this: Med. Chem. Commun., 2017, 8, 1414

Received 5th April 2017,

Accepted 3rd May 2017

DOI: $10.1039 / c 7 m d 00164 a$

rsc.li/medchemcomm

\section{Rational design and synthesis of novel phenylsulfonyl-benzamides as anti-prostate cancer agents $\dagger+\$$}

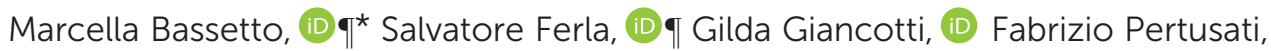 \\ Andrew D. Westwell, Andrea Brancale and Christopher McGuigan
}

\section{Introduction}

Prostate cancer (PC) is one of the major causes of male death worldwide, representing the second most common cancer in males. ${ }^{1}$ One of the main treatment strategies against this disease is currently represented by androgen deprivation: ${ }^{2}$ bicalutamide (1) and enzalutamide (2) are two non-steroidal androgen receptor (AR) antagonist drugs approved to treat PC (Fig. 1). Unfortunately, these anti-androgens tend to become ineffective in a few years' time due to adaptive mutations on the structure of the androgen receptor, which switch the function of these drugs from antagonist to agonist. ${ }^{2}$

One of the most common mutations found for bicalutamide is W741L in helix 12 of the receptor, ${ }^{3}$ which allows the protein to adopt its closed agonist conformation even in the presence of the antagonist: with this mutation, due to some residual structural flexibility in 1, ring B can bend to occupy an inner portion of the ligand-binding domain, thus allowing the closure of the receptor into its agonist conformation. Treatment with enzalutamide induces instead a F876L mutation in the AR, which also confers an antagonist to agonist switch in activity for the drug. ${ }^{4}$ Secondgeneration antiandrogen $\mathrm{ARN}-509$ (Fig. 1), which is now in

School of Pharmacy and Pharmaceutical Sciences, Redwood Building, King Edward VII Avenue, CF10 3NB, Cardiff, Wales, UK.

E-mail: BassettoM@cardiff.ac.uk

$\uparrow$ The authors declare no competing interests.

\$ Electronic supplementary information (ESI) available. See DOI: 10.1039/ c7md00164a

$\S$ This work is dedicated to the memory of Prof. Chris McGuigan, a great colleague and scientist, invaluable source of inspiration and love for research.

I These authors contributed equally to this work.
Phase III clinical trials, ${ }^{5}$ is also associated with the insurgence of resistance through the F876L mutation, ${ }^{6}$ while newgeneration AR inhibitor ODM-201 (Fig. 1), now in Phase III trials, has shown retained activity in the F876L AR mutant. ${ }^{7}$

Most non-steroidal antiandrogens are structurally characterised by two differently substituted aromatic rings, named ring A and ring B, connected by a linker, either linear (bicalutamide-like compounds) or cyclic (enzalutamide-like compounds) (Fig. 1). ${ }^{8} \quad$ Recently, a novel 4-(4-benzoylaminophenoxy) phenol antiandrogen scaffold (4), derived from the natural pigment curcumin, has been reported, in which a central phenyl group is acting as linker connecting two different aromatic rings. ${ }^{9}$

With the aim to rigidify the structure of bicalutamide and obtain new anticancer agents, we designed a novel molecular scaffold in which we replaced the flexible bicalutamide methyl-hydroxy-methylene linker with a rigid phenyl group, maintaining the two lateral aromatic rings commonly found in antiandrogen compounds (Fig. 2). Moreover, following an approach previously proven successful in our research group for increasing bicalutamide and enzalutamide anti-prostate cancer activity, ${ }^{10,11}$ different perfluoro groups were systematically inserted in aromatic $\operatorname{ring} B\left(R_{2}\right.$ substituent), replacing the classical 4-cyano substituent. The phenolic 4-hydroxy group of 4, previously reported as essential for AR antagonistic activity, ${ }^{9}$ was either maintained, in order to evaluate its importance for anti-prostate cancer effect, or replaced with a 3-trifluoromethyl group, as a means to incorporate and evaluate one of the most successful modifications we have previously found in other series of related compounds. ${ }^{10,11}$

Following this approach, a novel family of phenylsulfonylbenzamides was developed (Fig. 2), and their potential anti 

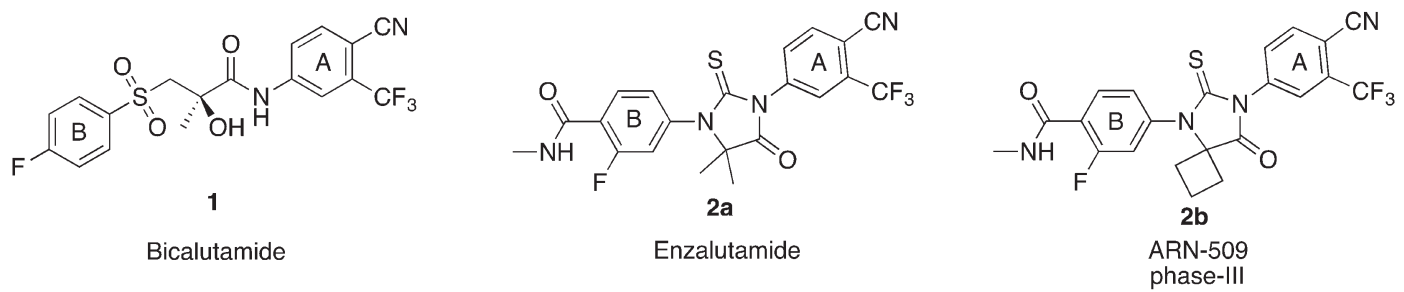

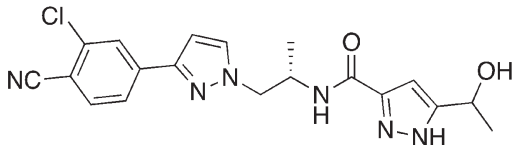

3

ODM-201

phase-II



4

Fig. 1 Structure of anti-androgen small molecules approved by FDA ( 1 and $2 a$ ), in clinical development for the treatment of PC (2b and 3) or in early-stage development (4). Two differently substituted aromatic rings, A and B, are connected by a linker.

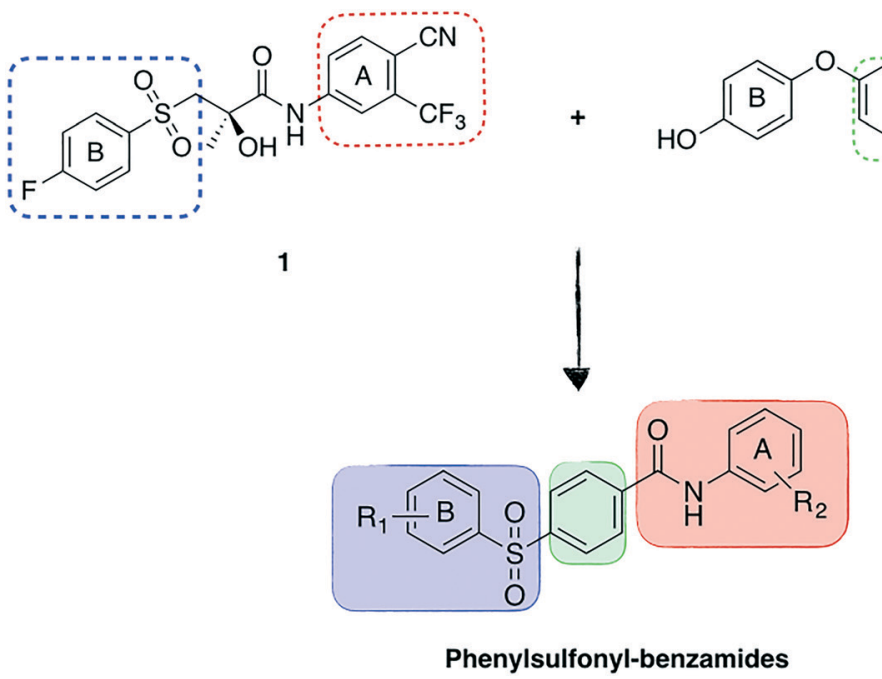

Fig. 2 Rational design of novel phenylsulfonyl-benzamide scaffold.

prostate cancer activity was evaluated in four different human prostate cancer cell lines, together with their cytotoxicity in the HEK293 cell line.

\section{Results and discussion}

\section{Molecular modelling}

A series of flexible alignment and molecular docking studies was performed to evaluate the proposed structural modifications. For these analyses, compound 13a, which maintains bicalutamide and enzalutamide aromatic substituents in ring A and is characterised by the fully oxidised sulfonyl linker, was chosen as representative for the novel series of compounds. In a previous work, ${ }^{10}$ we have extensively discussed and described the most likely binding mode of bicalutamide and enzalutamide in the homology model of the androgen receptor (AR) open antagonist conformation. In the present study, the predicted active conformation for the two drugs was kept rigid and a flexible alignment with newly designed compound 13a was performed using MOE2015.10, ${ }^{12}$ in order to evaluate its potential structural overlapping with the two active conformations. 13a aligns well with the binding pose of the parent compounds, with the newly introduced phenyl ring conferring the desired structural rigidity to lock the molecule in the active conformation (Fig. 3). Moreover, as reported in the ESIt (Table S1), the two conformations obtained for 13a show a large negative similarity score $F$, meaning that 13a shows a high shape and functional similarity to the two parent compounds. Finally, the $\mathrm{d} U$ value found for both alignments is lower than $1 \mathrm{kcal} \mathrm{mol}^{-1}$, meaning that the obtained conformations are energetically favoured. The better (more negative) similarity score obtained for the alignment with enzalutamide is a further confirmation that the introduction of the phenyl linker rigidifies the novel structure, potentially locking the molecule in an active conformation.

Fig. 4A shows the docking results found for 13a in the ligand binding domain (LBD) of the open AR homology model, obtained using Plants docking software. ${ }^{13}$ The extra phenyl ring, as observed in the flexible alignment results, maintains the molecule in a rigid extended conformation that entirely 
A)

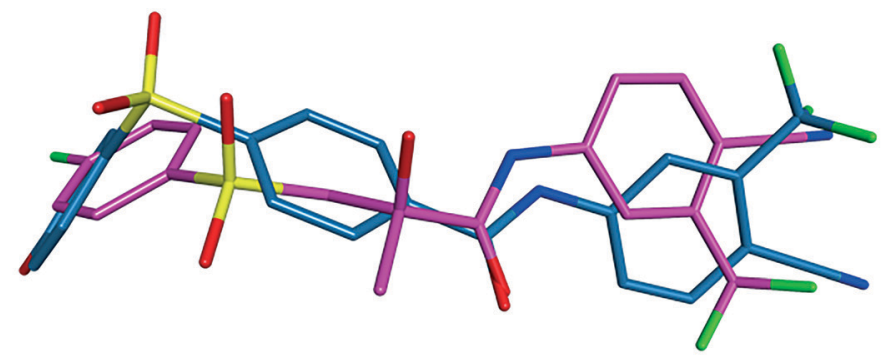

dU: 0.000

S: -95.4702

F: -136.9531

B)

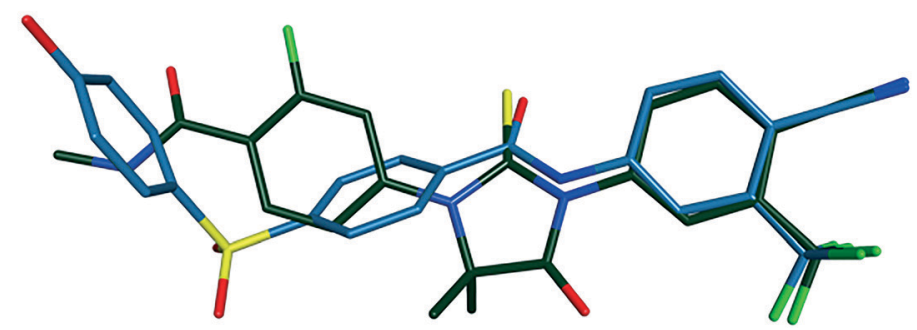

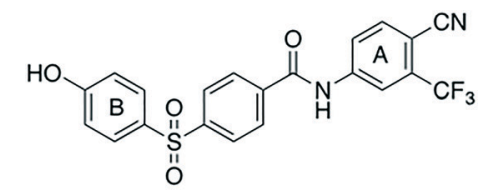

$13 a$

dU: 0.000

S: -108.1487

F: -149.6317

Fig. 3 Flexible alignment between 13a (carbon atoms in light blue) with the predicted active binding conformations of bicalutamide (A-carbon atoms in purple) and enzalutamide (B-carbon atoms in dark green).

occupies the binding site, similarly to bicalutamide and enzalutamide. The phenolic hydroxyl group is facing outside the binding groove, therefore its replacement with other functions is predicted to be tolerated. Docking results for 13a in the bicalutamide-resistant AR mutant W741L crystal structure (PDB ID 1Z95), ${ }^{3}$ which corresponds to the closed active conformation of the receptor, indicate that, due to its increased rigidity, the novel molecule cannot fit the AR antagonist conformation, as highlighted by the steric clashes between 13a and helix 12 (Fig. 4B). These results suggest that the novel scaffold could maintain its antagonist activity also in the presence of the $\mathrm{W} 741 \mathrm{~L}$ mutation.

\section{Chemistry}

Scheme 1 reports the three-step synthetic pathway developed and optimised for the preparation of the newly designed compounds.

In the first step a copper catalysed C-S coupling reaction was used for the preparation of intermediates 8-9. The conditions for this step were optimised from a reported procedure. ${ }^{14}$ After 12 hours reflux as in the published protocol, 4-iodobenzoic acid 7 was still present in the reaction mixture. No difference could be observed with a prolonged reaction time, while total consumption of starting material 7 was finally achieved by doubling the equivalents of thiophenol and base. The new conditions were applied to react differently substituted thiophenols 5-6 with 4-iodobenzoic acid 7 for 24 hours in water, giving the desired different substituted phenylthio-benzoic acids in an almost quantitative yield. Amides 11a-i and 12a, $h$ were obtained reacting the correspond- ing anilines 10a-i with 8 and 9 in the presence of thionyl chloride in DMA, modifying a literature procedure. ${ }^{15}$ In the last step, sulphur derivatives $11 a-i$ and $12 a, h$ were oxidized to the corresponding sulfones 13a-i and 15a, $\mathbf{h}$ using mCPBA at $25{ }^{\circ} \mathrm{C} .{ }^{10}$ In two cases, the corresponding product of sulphur partial oxidation was also isolated (14e and 14i). These two sulfoxides were characterised by low solubility in the reaction solvent, therefore complete oxidation to the corresponding sulfone was much slower and did not go to completion within the reaction time. Using this synthetic approach, 24 novel phenylsulfonyl-benzamides derivatives were prepared, purified and fully characterised.

Standard bicalutamide and enzalutamide were also prepared following reported procedures. ${ }^{10}$

\section{In vitro $2 \mathrm{D}$ monolayer antiproliferative assay}

All new compounds along with the two standards were tested for their ability to inhibit survival and/or cell proliferation in four human prostate cancer cell lines: LNCaP, 22Rv1, VCaP, androgen-sensitive cell lines, and DU145, a non hormonesensitive cell line. Table 1 shows the antiproliferative results for each single cell line (absolute $\mathrm{IC}_{50}$ in $\mu \mathrm{M}$ ), along with the overall effect in the four cell lines reported as geometric mean. Activity found for bicalutamide and enzalutamide is consistent with previously published data for some of these specific cell lines ${ }^{16-18}$ confirming the reliability of the assay.

Considering their overall profile in the four cell lines (geometric mean), almost all the new derivatives performed significantly better than bicalutamide, improving its effect up to 16-fold. The new inhibitors showed concentration-dependent 


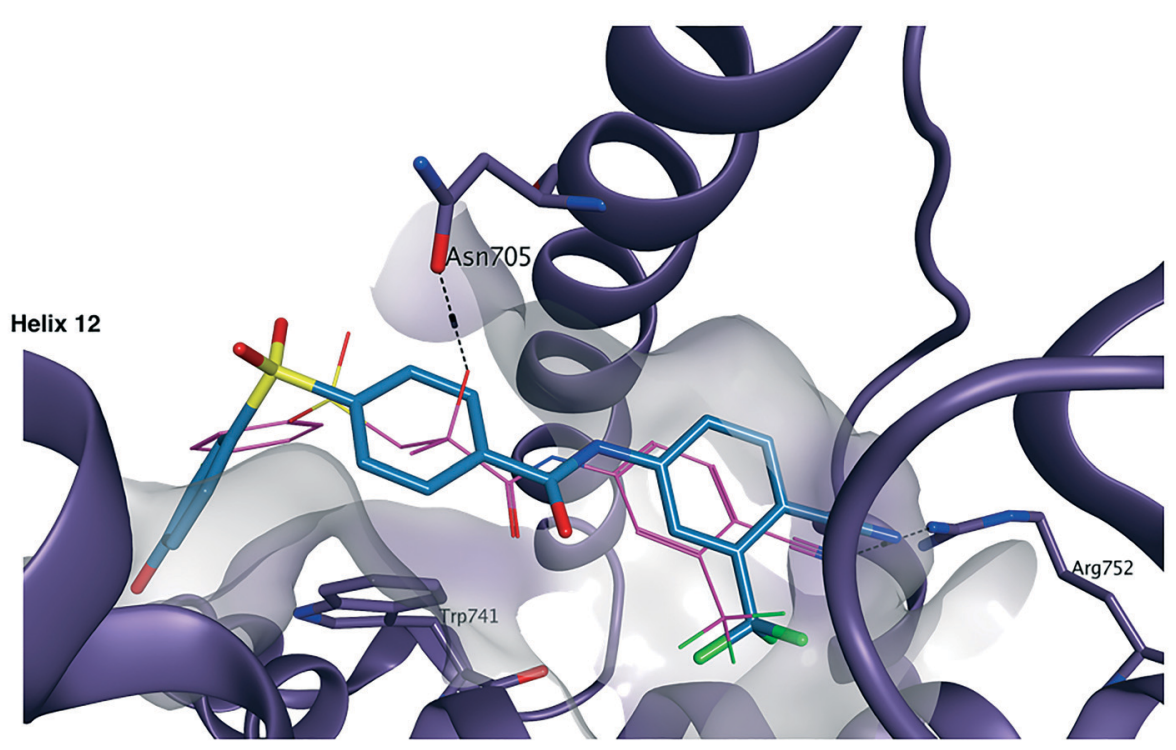

B)

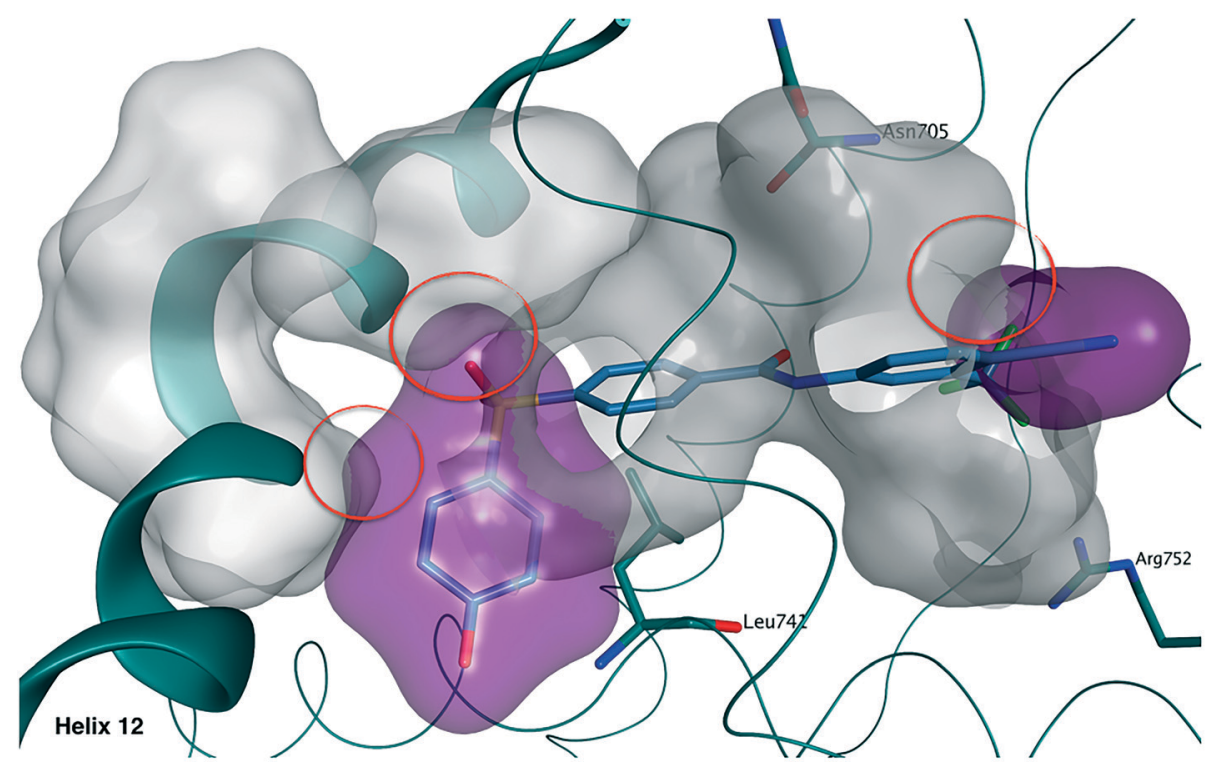

Fig. 4 A) Predicted binding mode of 13a (carbon atoms in light blue) in the AR homology model. ${ }^{10}$ The compound occupies the LBD in a similar way to bicalutamide (carbon atoms in purple). B) 13a in the bicalutamide-W741l AR closed conformation crystal structure 1295 (B). ${ }^{3}$ The compound shows major clashes (purple surface), highlighted by red circles, with the protein surface (in grey) of the closed AR structure, especially with helix 12 , indicating that it might impede the AR closure even in the presence of adaptive mutations.

activity with mean $\mathrm{IC}_{50}$ values ranging from $3.2 \mu \mathrm{M}$ to $>100$ $\mu \mathrm{M}$. Compared with standard enzalutamide, antiproliferative effect was improved up to 8-fold.

Substituent $R_{1}$ in ring $B$ does not seem to influence the anticancer profile of the scaffold, as no substantial differences were found between the $\mathrm{OH}$ group in the para position or the $\mathrm{CF}_{3}$ in meta (i.e. 11a vs. 12a). This finding is in accordance with the proposed binding mode for 13a, in which the $\mathrm{R}_{1}$ substituent is facing outside the AR binding domain without making specific interactions.

In both our previous work on bicalutamide derivatives and in another published work on analogues of SARMs (selective androgen receptor modulators), ${ }^{10,16}$ thioether compounds (X $=\mathrm{S}$ ) are associated with better antiproliferative activities than the corresponding sulfones $\left(\mathrm{X}=\mathrm{SO}_{2}\right)$, with a decrease of effect in the sulfones up to 8-fold. In the new scaffold presented in this work, no particular differences can be observed between thioether and sulfone compounds, with retained activity in most sulfones (i.e. 11a vs. 13a) and only a small increase of $\mathrm{IC}_{50}$ values up to 2 -fold in few cases (i.e. 11c vs. 13c). The partially oxidized derivatives $(\mathrm{X}=\mathrm{SO})$ retain the effect of the corresponding thioethers and sulfones (i.e. 11e and 13e vs. 14e).

Removal of the $\mathrm{CN}$ or the $\mathrm{NO}_{2}$ group in the para position of ring $\mathrm{B}$ is associated with retained activity, as found for 
<smiles>O=C(O)c1ccc(I)cc1</smiles>

$5,8,11,13,14 \mathrm{R}_{1}=4-\mathrm{OH}$

$6,9,12,15 \mathrm{R}_{1}=3-\mathrm{CF}_{3}$

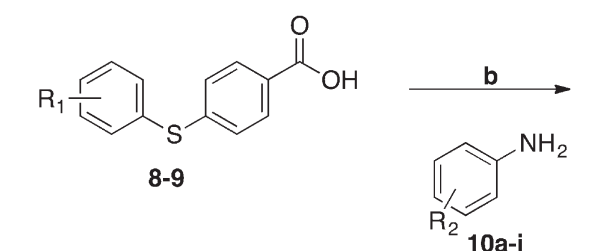

a $\mathrm{R}_{2}=4-\mathrm{CN}, 3-\mathrm{CF}_{3}$

b $\mathrm{R}_{2}=4-\mathrm{NO}_{2}, 3-\mathrm{CF}_{3}$

c $\mathrm{R}_{2}=3,5-\mathrm{CF}_{3}$

d $\mathrm{R}_{2}=2,5-\mathrm{CF}_{3}$

e $\mathrm{R}_{2}=4-\mathrm{SF}_{5}$

f $R_{2}=3-S_{5}$

g $\mathrm{R}_{2}=4-\mathrm{CF}_{3}$

h R $\mathrm{R}_{2}=3-\mathrm{CF}_{3}$

i $\mathrm{R}_{2}=2-\mathrm{CF}_{3}$<smiles></smiles><smiles></smiles>

13a-i $\mathrm{X}=\mathrm{SO}_{2}$

$14 \mathrm{e}, \mathrm{i} X=\mathrm{SO}$

$15 \mathrm{a}, \mathrm{h} \mathrm{X}=\mathrm{SO}_{2}$

Scheme 1 Synthetic pathway for the synthesis of phenylsulfonyl-benzamides analogues. Reagents and conditions: (a) $\mathrm{KOH}, \mathrm{Cu}, \mathrm{H}_{2} \mathrm{O}, \mathrm{reflux}, 24 \mathrm{~h}$; (b) 8-9 (1 equiv.), SOCl 2 (1.2 equiv.), DMA, -15 to $-10{ }^{\circ} \mathrm{C}$ to rt. After $1 \mathrm{~h} 10 \mathrm{a}-\mathrm{i}$ (1 equiv.) dissolved in DMA is added, $r$, on; (c) mCPBA (1.4 equiv.), DCM, rt, on.

mono $3-\mathrm{CF}_{3}$ derivative $\mathbf{1 1 h}$. Moving the trifluoromethyl group from meta to para position does not have a significant effect on activity (i.e. $11 \mathrm{~h} v$ s. $\mathbf{1 4 g}$ ), whereas the ortho substitution is characterized by a 3 -fold reduction of overall effect in the four cell lines (i.e. 11h vs. 11i). Replacing the trifluoromethyl group with a bigger $-\mathrm{SF}_{5}$ moiety in para or meta position is associated with a mildly improved activity profile (i.e. $11 \mathrm{~g} v$ s. 14e).

Addition of an extra $\mathrm{CF}_{3}$ in meta $\left(\mathrm{R}_{2}=3,5-\mathrm{CF}_{3}\right)$, gives the best results in terms of overall activity in the four cell lines

Table 1 Antiproliferative activity for the novel phenylsulfonyl-benzamide derivatives. All data are mean values from triplicate experiments, with standard deviations of $\pm 10 \%$ of the value quoted unless otherwise stated. Compound $15 \mathrm{~h}$ showed solubility issues

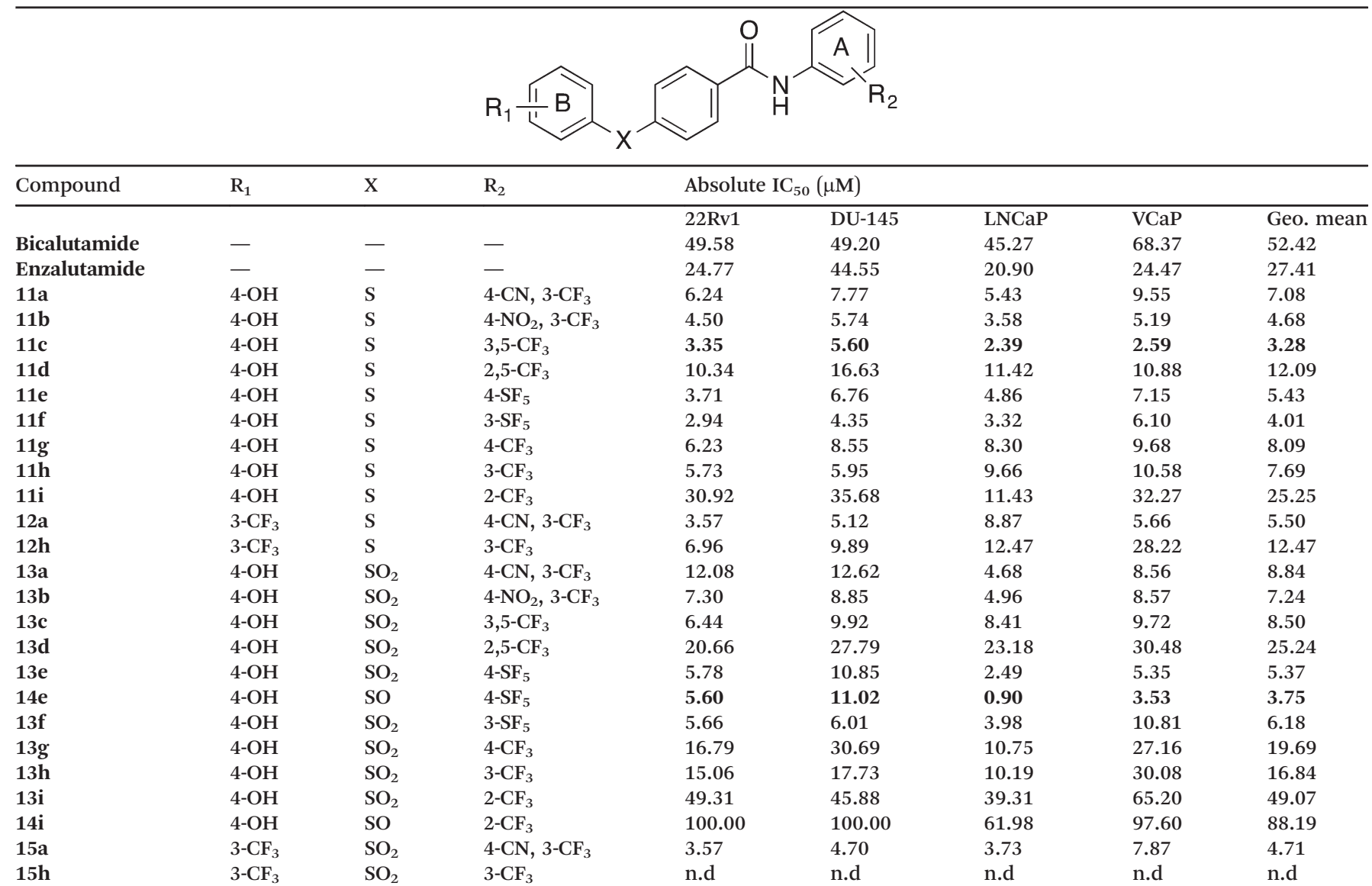




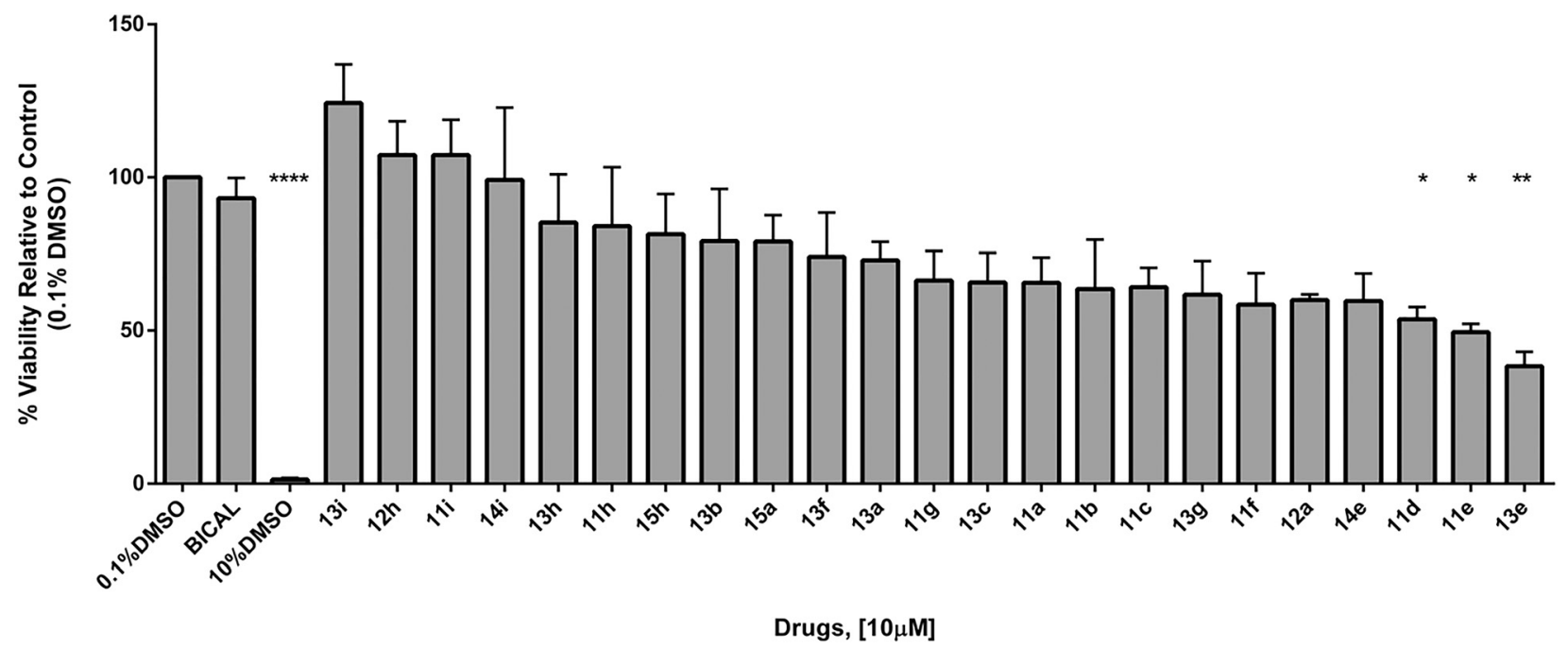

Fig. 5 HEK293 human embryonic kidney cells were treated at $70 \%$ confluence with negative controls (0.1\% DMSO, BICAL at $10 \mu M)$, positive control (10\% DMSO) and compounds at $10 \mu \mathrm{M}$ concentration for 24 hours. $20 \mu \mathrm{l}$ of Cell Titer Blue reagent (Promega) was added to each well containing $100 \mu \mathrm{l}$ media and cell were incubated for $2 \mathrm{~h}$ at $37{ }^{\circ} \mathrm{C} 5 \% \mathrm{CO}_{2}$. Fluorescence was measured at 560/590 nm using a CLARIOstar luminescence plate reader (BMG Labtech). $N=4, n=6$ for controls, $n=3$ for drugs. Errors are calculated as standard error of the mean. $*=$ $p$-value $<0.05, * *=p$-value $<0.01, * * *=p$-value $<0.001 * * * *=p$-value $<0.0001$ between bicalutamide and compounds.

(i.e. 11a and 13h vs. 14c), as we had similarly observed in our previous research efforts. ${ }^{10}$ Also in this case, moving one trifluoromethyl group from meta to ortho position is associated with activity reduction (i.e. 11c vs. 11d).

Examining each individual cell line, most of the new compounds showed the best results in the cell lines that highly express the androgen receptor. Among them, LNCaP cells were found to be the most sensitive to the new derivatives, with different compounds active at low $\mu \mathrm{M}$ concentrations and one reaching a sub $\mu \mathrm{M} \mathrm{IC}_{50}$ value $(\mathbf{1 4 e})$. These data suggest that the newly prepared compounds are likely to retain an antagonistic effect on the androgen receptor. An interesting activity profile was also found in the DU-145 cell line (the least sensitive to our new molecules), which expresses low levels of the androgen receptor ${ }^{19}$ and is insensitive to androgen activity. The same effect can be observed also for parent bicalutamide (similar $\mathrm{IC}_{50}$ values across the four cell lines), suggesting that besides its canonical anti-androgen receptor action, a different antiproliferative mechanism could also be involved. The new phenylsulfonyl-benzamides, in addition to potentially increase the antagonistic activity on the AR, might also enhance the potential off target effect, even though in some cases they seem more selective for androgen-sensitive cell lines than bicalutamide (i.e. 13e, 14e, 13g). As a general observation, the improved antiproliferative activity of the novel compounds should be regarded as the result of the whole structure of each single derivative, since the data found appear to be a combination of the simultaneous effects of the substituent in ring $\mathrm{B}$ and linker $\mathrm{X}$.

\section{Cytotoxicity assay}

A Cell Titer Blue viability assay was performed in the human embryonic kidney cell line HEK293, in order to evaluate the cytotoxicity profile of the newly prepared compounds. Fig. 5 shows the reduction of cell viability caused by the novel analogues and standard bicalutamide at a fixed concentration of $10 \mu \mathrm{M}$. Interestingly, as reported in the graph, only three compounds (11d, 11e and 13e) significantly reduced cell viability if compared to bicalutamide (statistical significance), and only one of them (13e) killed more than $50 \%$ of cells. Due to their higher cytotoxicity in comparison with bicalutamide, the antiproliferative effect of these three compounds in the prostate cancer cell lines could also be a consequence of their intrinsic toxicity, besides the canonical AR antagonist activity. Comparing the rest of the compounds with bicalutamide, even if some of them are characterized by an increased cytotoxicity, cell viability reduction is not statistically significant and less than $40 \%$ in most cases, therefore the observed cytotoxicity is comparable to the one found for bicalutamide. Moreover, it is worth to note that bicalutamide reaches its antiproliferative $\mathrm{IC}_{50}$ at $50 \mu \mathrm{M}$, therefore it would not show reduction of cell viability at $10 \mu \mathrm{M}$. On the contrary, most of our new compounds showed antiproliferative activity in the four prostate cancer cell lines at a low $\mu \mathrm{M}$ concentration $(<10 \mu \mathrm{M})$, and even if some of them show a reduction of cell viability by $35-40 \%$, their anticancer therapeutic window is still wide enough for consideration as a potential treatment for prostate cancer. In particular, compounds $11 \mathrm{~h}, \mathbf{1 2 h}, \mathbf{1 3 b}$ and $13 \mathrm{~h}$ are associated with a promising antiproliferative/cytotoxicity profile, with an antiproliferative effect much better than bicalutamide $(<16$ $\mu \mathrm{M})$, and with the same cytotoxicity of bicalutamide at $10 \mu \mathrm{M}$ (none or minimal reduction of cell viability).

\section{Conclusions}

A novel family of phenylsulfonyl-benzamides was designed and synthesised after rational modification of the structure 
of antiandrogen drug bicalutamide, and many of the novel derivatives prepared show improved anti-prostate cancer properties. In particular, most of the new compounds display significantly improved anticancer activities in four different prostate cancer cell lines, with a cell viability reduction effect comparable to the one found for bicalutamide. Differently from previously reported SARMs, no significant difference in antiproliferative activity has been observed between our novel thioether and sulfone derivatives, representing a promising feature for future metabolic stability considerations. Within the 24 novel analogues evaluated, $11 \mathrm{~h}, 12 \mathrm{~h}, 13 \mathrm{~b}$ and $13 \mathrm{~h}$ have been selected as candidates for further investigations, due to their improved anticancer properties and their cytotoxicity profiles. With the aim to identify a pre-clinical candidate, these compounds will be studied for their binding to the AR, their potential off target effect and their pharmacokinetic properties.

\section{Experimental}

All chemistry, biology and molecular modelling experimental procedures, along with compound characterisation, are fully described in the ESI. + All final compounds were purified by column chromatography or recrystallization and fully characterised by NMR $\left({ }^{1} \mathrm{H},{ }^{13} \mathrm{C},{ }^{19} \mathrm{~F}\right)$ and LRMS. All final compounds were found to be $>95 \%$ pure by HPLC.

\section{Acknowledgements}

The authors would like to thank Oncotest (Freiburg, Germany) for provision of human prostate cancer cell line testing as an outsourced service. The Welsh Government is acknowledged for funding (A4B-Academic Expertise for Business grant).

\section{References}

1 www.cancerresearchuk.gov (accessed April 3, 2017).

2 J. Ferlay, H.-R. Shin, F. Bray, D. Forman, C. Mathers and D. M. Parkin, Int. J. Cancer, 2010, 127, 2893-2917.

3 C. E. Bohl, W. Gao, D. D. Miller, C. E. Bell and J. T. Dalton, Proc. Natl. Acad. Sci. U. S. A., 2005, 102, 6201-6206.

4 M. Korpal, J. M. Korn, X. Gao, D. P. Rakiec, D. A. Ruddy, S. Doshi, J. Yuan, S. G. Kovats, S. Kim, V. G. Cooke, J. E. Monahan, F. Stegmeier, T. M. Roberts, W. R. Sellers, W. Zhou and P. Zhu, Cancer Discovery, 2013, 3, 1030-1043.

5 N. J. Clegg, J. Wongvipat, J. D. Joseph, C. Tran, S. Ouk, A. Dilhas, Y. Chen, K. Grillot, E. D. Bischoff, L. Cai, A. Aparicio,
S. Dorow, V. Arora, G. Shao, J. Qian, H. Zhao, G. Yang, C. Cao, J. Sensintaffar, T. Wasielewska, M. R. Herbert, C. Bonnefous, B. Darimont, H. I. Scher, P. Smith-Jones, M. Klang, N. D. Smith, E. De Stanchina, N. Wu, O. Ouerfelli, P. J. Rix, R. A. Heyman, M. E. Jung, C. L. Sawyers and J. H. Hager, Cancer Res., 2012, 72, 1494-1503.

6 J. D. Joseph, N. Lu, J. Qian, J. Sensintaffar, G. Shao, D. Brigham, M. Moon, E. C. Maneval, I. Chen, B. Darimont and J. H. Hager, Cancer Discovery, 2013, 3, 1020-1029.

7 K. Fizazi, L. Albiges, Y. Loirot and C. Massard, Expert Rev. Anticancer Ther., 2015, 15, 1007-1017.

8 C. Ferroni, A. Pepe, Y. Sang Kim, S. Lee, A. Guerrini, M. D. Parenti, A. Tesei, A. Zamagni, M. Cortesi, N. Zaffaroni, M. De Cesare, G. L. Beretta, J. B. Trepel, S. V. Malhotra and G. Varchi, J. Med. Chem., 2017, 60, 3082-3093.

9 A. Yamada, S. Fujii, S. Mori and H. Kagechika, ACS Med. Chem. Lett., 2013, 4, 937-941.

10 M. Bassetto, S. Ferla, F. Pertusati, S. Kandil, A. D. Westwell, A. Brancale and C. McGuigan, Eur. J. Med. Chem., 2016, 118, 230-243.

11 S. Ferla, M. Bassetto, F. Pertusati, S. Kandil, A. D. Westwell, A. Brancale and C. McGuigan, Bioorg. Med. Chem. Lett., 2016, 26, 3636-3640.

12 Molecular Operating Environment (MOE 2015.10); Chemical Computing Group, Inc.: Montreal, Quebec, Canada; URL http://www.chemcomp.com.

13 O. Korb, T. Stützle and T. E. Exner, Swarm Intell., 2007, 1, 115-134.

14 L. Costantino, A. M. Ferrari, M. C. Gamberini and G. Rastelli, Bioorg. Med. Chem., 2002, 10, 3923-3931.

15 Y. He, D. Yin, M. Perera, L. Kirkovsky, N. Stourman, W. Li, J. T. Dalton and D. D. Miller, Eur. J. Med. Chem., 2002, 37, 619-634.

16 D. J. Hwang, J. Yang, H. Xu, I. M. Rakov, M. L. Mohler, J. T. Dalton and D. D. Miller, Bioorg. Med. Chem., 2006, 14, 6525-6538.

17 A. Colabufo, V. Pagliarulo, F. Berardi, M. Contino, C. Inglese, M. Niso, P. Ancona, G. Albo, A. Pagliarulo and R. Perrone, Eur. J. Pharmacol., 2008, 601, 38-42.

18 H. Kuruma, H. Matsumoto, M. Shiota, J. Bishpo, F. Lamoureux, C. Thomas, D. Briere, G. Los, M. Gleave, A. Fanjui and A. Zoubeidi, Mol. Cancer Ther., 2013, 12, 567-576.

19 F. Alimirah, J. Chen, Z. Basrawala, H. Xin and D. Choubey, FEBS Lett., 2006, 580, 2294-2300. 